\title{
A Lagrangian approach to identifying vortex pinch-off
}

\author{
Clara O'Farrell ${ }^{1}$ and John O. Dabiri ${ }^{2}$ \\ ${ }^{1}$ Control and Dynamical Systems, California Institute of Technology, Pasadena, California 91125, USA \\ ${ }^{2}$ Graduate Aeronautical Laboratories and Bioengineering, California Institute of Technology, \\ Pasadena, California 91125, USA
}

(Received 13 August 2009; accepted 28 November 2009; published online 5 January 2010)

\begin{abstract}
A criterion for identifying vortex ring pinch-off based on the Lagrangian coherent structures (LCSs) in the flow is proposed and demonstrated for a piston-cylinder arrangement with a piston stroke to diameter $(L / D)$ ratio of $\approx 12$. It is found that the appearance of a new disconnected LCS and the termination of the original LCS are indicative of the initiation of vortex pinch-off. The subsequent growth of new LCSs, which tend to roll into spirals, indicates the formation of new vortex cores in the trailing shear layer. Using this criterion, the formation number is found to be $4.1 \pm 0.1$, which is consistent with the predicted formation number of $\approx 4$ of Gharib et al. [J. Fluid Mech. 360, 121 (1998)]. The results obtained using the proposed LCS criterion are compared with those obtained using the circulation criterion of Gharib et al. and are found to be in excellent agreement. The LCS approach is also compared against other metrics, both Lagrangian and Eulerian, and is found to yield insight into the pinch-off process that these do not. Furthermore, the LCS analysis reveals a consistent pattern of coalescing or "pairing" of adjacent vortices in the trailing shear layer, a process which has been extensively documented in circular jets. Given that LCSs are objective and insensitive to local errors in the velocity field, the proposed criterion has the potential to be a robust tool for pinch-off identification. In particular, it may prove useful in the study of unsteady and low Reynolds number flows, where conventional methods based on vorticity prove difficult to use.
\end{abstract}

(C) 2010 American Institute of Physics. [doi:10.1063/1.3275499]

The formation of axisymmetric vortex rings is a widely occurring phenomenon both in nature and in industry. It is known that these vortex rings cannot grow indefinitely, but rather there is a physical limit to their size. ${ }^{1}$ Beyond this limit, vortex rings do not grow any further but "pinch off," and a trailing jet forms behind them. This limit implies the existence of an optimum vortex size: for optimum momentum transfer, rings must be made as large as possible while avoiding pinch-off. ${ }^{2}$ This optimum has important implications for natural and engineering flows, and hence vortex ring pinch-off has been extensively studied, principally by means of the vorticity field. However, in the more complex naturally occurring flows, the vorticity field tends to break down and diffuse, and existing criteria prove insufficient for robustly identifying pinch-off. In this paper we propose a criterion for pinchoff identification, based on Lagrangian coherent structures (LCSs), which could provide further insight into the structure of these complex natural flows. The criterion is demonstrated for a laboratory-generated vortex ring, and it is found to be in good agreement with the established criterion based on circulation.

\section{INTRODUCTION}

Vortex rings are a recurring feature of key importance in a wide variety of biological ${ }^{3-5}$ and engineering flows ${ }^{6-8}$ and have hence been the subject of various experimental and computational studies. In particular, vortex rings generated in the laboratory using a piston-cylinder apparatus have been the subject of intensive study. ${ }^{1,-11}$ In these experiments, a fluid column of length $L$ is pushed through a circular aperture or nozzle of diameter $D$ by a piston moving with prescribed velocity. A boundary layer of vorticity forms inside the cylinder as the piston moves, which then separates at the edge of the aperture and rolls up into a vortex ring.

Gharib et al. ${ }^{1}$ found that vortex rings cannot grow indefinitely, but rather there is a physical limit to their size. As the vortex ring evolves, it accepts vorticity flux from the pistoncylinder apparatus which fuels its growth. When the ratio of piston stroke to diameter $(L / D)$ exceeds $\approx 4$, Gharib et al. ${ }^{1}$ found that the vortex rings rejected any additional vorticity flux, which instead formed a wake of Kelvin-Helmholtztype vortices trailing the ring. They termed the disconnection of the velocity and vorticity fields of the vortex ring from the trailing jet "vortex pinch-off," and the $L / D$ ratio at which the disconnection was initiated the "formation number" $(F)$.

In order to determine the formation number, Gharib et $a l .{ }^{1}$ measured the total circulation discharged by the pistoncylinder apparatus as well as the circulation of the vortex ring once it had separated from the trailing shear layer as a function of the formation time $\left(L / D\right.$, where $L=\bar{U}_{p} t$ is the length of the fluid column already ejected and $\bar{U}_{p}$ is the running average of the piston speed). The authors reasoned that only the vorticity that had emanated from the cylinder before the vortex had ceased to accept vorticity (i.e., before the onset of pinch-off) could accumulate in the vortex ring. Therefore, they compared the time histories of the two circulations to determine the $L / D$ at which the total circulation emanating from the cylinder equaled the maximum circula- 
tion of the vortex ring, and termed this ratio the formation number.

Using this method, Gharib et al., ${ }^{1}$ Krueger et al., ${ }^{12}$ Dabiri and Gharib, ${ }^{13}$ and others were able to identify the formation number for a variety of flow conditions using the pistoncylinder configuration. Others ${ }^{14-16}$ have observed vortex pinch-off in the laboratory in other vortex-shedding configurations. Furthermore, vortex pinch-off has been observed in biological flows such as that through the mitral valve in the human heart ${ }^{5}$ and the flow generated by jetting squid ${ }^{4}$ and jellyfish. $^{3}$

The method of Gharib et al. ${ }^{1}$ poses several challenges, however. First, determining the final circulation of the vortex ring requires that the ring be distinguishable from its trailing jet from vorticity contours. Since such a distinction is not possible until the vortex has advected away from the wake, it requires observation of the vortex ring's evolution after the initiation of pinch-off. In unsteady flows, vortex breakdown can obscure these long-term observations. Second, even if one has the capability to record the vortex evolution for these long times, it is essential that the vorticity field not diffuse and the vortex not distort by interaction with other structures in the flow, both of which are common processes in biological flows. Finally, as Bartol et al. ${ }^{4}$ remark, in low Reynolds number flows, it can be difficult to discern whether or not the elongated nature of some naturally occurring vortex rings represents pinch-off that has been obscured by viscous diffusion.

We propose an alternate method for identifying vortex ring pinch-off using LCSs. LCSs are finite-time invariant manifolds, akin to the invariant manifolds in timeindependent flows, which act as separatrices between regions of qualitatively different flow. ${ }^{11}$ Moreover, changes in the LCS structure have been shown to correspond to changes in vortex structure by Green $e t a l .{ }^{17}$ Since the pinch-off process involves the separation of distinct vorticity-carrying regions of the flow (i.e., the vortex ring and trailing jet, respectively), we propose that LCSs are an effective tool for quantifying the process.

We consider a starting jet flow with an $L / D$ ratio of approximately 12 . Using LCSs, we are able to identify the onset of pinch-off without requiring that the circulation in the vortex ring be measured after pinch-off. Furthermore, the LCS approach provides insight into the structure and dynamics of the trailing shear layer which analysis of the vorticity alone, or other traditional metrics, overlooks.

\section{METHODS}

\section{A. Vortex ring generation}

Vortex rings were generated in a water tank using the piston-cylinder arrangement described by Dabiri and Gharib. ${ }^{18}$ However, whereas Dabiri and Gharib ${ }^{18}$ considered low $L / D$ ratios only $(L / D=2,4)$, we consider a case where $L / D \approx 12$. Flow from a constant-head tank was allowed to drive a piston through a hollow cylinder of internal diameter $D=2.54 \mathrm{~cm}$ submerged in the water tank. A column of fluid of length $L \approx 30.5 \mathrm{~cm}$ was impulsively ejected from the sharp-edged nozzle by the motion of the piston, and rolled up into a vortex ring which propagated through the surrounding fluid owing to its self-induced velocity.

The resulting velocity field was recorded using digital particle image velocimetry (DPIV), as described by Dabiri and Gharib ${ }^{18}$ and Shadden et al. ${ }^{11}$ A pulsed Nd:yttrium aluminum garnet laser sheet was used to illuminate a symmetry plane of the axisymmetric flow, and the flow was seeded with nominally $13 \mu \mathrm{m}$ neutrally buoyant glass spheres which scattered incident light from the laser sheet onto a charge coupled device camera whose image plane was positioned parallel to the sheet. The test section measured approximately $12 \mathrm{~cm}(4.7 D)$ radially and $20 \mathrm{~cm}(7.9 D)$ axially, and the resolution of the captured images was approximately $0.19 \times 0.19 \mathrm{~mm}^{2}$. The resulting images were interrogated using the method of Willert and Gharib ${ }^{19}$ with a separation of $18 \mathrm{~ms}$ between frames and an interrogation window size of $32 \times 32$ pixels with a $50 \%$ overlap. The velocity and vorticity fields were calculated using an in-house code, and the resolution for both was approximately $3 \times 3 \mathrm{~mm}^{2}$ $(0.13 \times 0.13 D)$ with an uncertainty of $1 \%$ and $3 \%$, respectively.

\section{B. Lagrangian coherent structures}

Haller and co-worker ${ }^{20-23}$ demonstrated the existence of "finite-time invariant manifolds" in time-dependent, aperiodic systems. These manifolds, which Haller termed LCSs, are analogous to the stable and unstable manifolds in timeindependent systems. Just as two points straddling the stable manifold of a hyperbolic fixed point in a time-independent system will diverge exponentially in forward time, points straddling a repelling LCS will diverge in forward time. Likewise, points straddling an attracting LCS will diverge in backward time, a behavior akin to the exponential divergence in backward time of points straddling the unstable manifold of a hyperbolic fixed point. Like stable and unstable manifolds, LCSs act as separatrices between kinematically distinct regions in the flow. ${ }^{11}$

To identify the LCS in the DPIV velocity fields we first computed the finite-time Lyapunov exponent (FTLE) field at every time step. Given a flow map $\phi_{t_{0}}^{t_{0}+T}: \mathbf{x}\left(t_{0}\right) \mapsto \mathbf{x}\left(t_{0}+T\right)$ that maps fluid particles from their initial position at $t_{0}$ to their position a time $T$ later, the FTLE is given by

$$
\sigma_{t_{0}}^{T}=\frac{1}{|T|} \ln \left\|\frac{d \mathbf{x}\left(t_{0}+T\right)}{d \mathbf{x}\left(t_{0}\right)}\right\|,
$$

where $d \mathbf{x}\left(t_{0}+T\right) / d \mathbf{x}\left(t_{0}\right)$ is the deformation tensor. $\sigma_{t_{0}}^{T}$ measures the linearized divergence of trajectories starting near $\mathbf{x}\left(t_{0}\right)$ over a time interval $T$. T may be positive (indicating that the FTLE field is calculated by integrating trajectories in forward time) or negative (indicating integration in backward time).

The FTLE field was computed on a Cartesian grid with a resolution of $0.3 \times 0.3 \mathrm{~mm}^{2}(0.01 \times 0.01 D)$. At each time $t$, each point on the grid was advected by the flow by numerically integrating the velocity field data for the integration time $T$. A fourth-order Runge-Kutta scheme was used for the numerical integration, and bilinear interpolation was used 
whenever interpolation of the velocity data was required. The deformation tensor was then computed at each point on the grid using central differencing with neighboring grid points. Finally, the FTLE field was computed by evaluating Eq. (1) at each point on the grid.

Regions of maximum fluid particle separation (for $T>0$ ) or maximum fluid particle attraction (for $T<0$ ) produce local maximizing curves known as "ridges" in the FTLE field. ${ }^{21}$ However, ridges in the FTLE field need not necessarily represent hyperbolic LCSs as they may correspond to lines of high shear. In order to confirm that a ridge is indeed a hyperbolic LCS, the sign of the instantaneous strain rate normal to the ridge must be checked. ${ }^{23}$ Ridges in the forward-time $(T>0)$ FTLE field where the strain rate normal to the ridge is positive (particle stretching away from the ridge) represent a repelling LCS. Conversely, ridges in the backward-time $(T<0)$ FTLE field where the strain rate normal to the ridge is negative (particle attraction toward the ridge) represent an attracting LCS. ${ }^{23}$

Ridges in the FTLE field were extracted using the method described by Mathur et al. ${ }^{24}$ We identified regions around the ridges where the gradient of the FTLE $\left(\nabla \sigma_{t_{0}}^{T}(\mathbf{x})\right)$ exceeded a set threshold and used a two-dimensional gradient climb within these regions to identify the locus of points on the ridge. A point was considered to be on the ridge when the Hessian of the FTLE $\left(\nabla^{2} \sigma_{t_{0}}^{T}(\mathbf{x})\right)$ had at least one negative eigenvalue and "no appreciable change" ${ }^{24}$ was observed in the angle between the eigenvector corresponding to the eigenvalue of $\nabla^{2} \sigma_{t_{0}}^{T}(\mathbf{x})$ with the smallest norm and $\nabla \sigma_{t_{0}}^{T}(\mathbf{x})$.

For each point on a ridge, we approximated the unit normal to the ridge by $\mathbf{n}=\nabla \sigma_{t_{0}}^{T}(\mathbf{x}) /\left\|\nabla \sigma_{t_{0}}^{T}(\mathbf{x})\right\|$ and computed the instantaneous rate of strain normal to ridge as $\langle\mathbf{n}, \mathbf{S n}\rangle$, where the rate of strain tensor $\mathbf{S}$ is the symmetric part of the velocity gradient tensor $\nabla \mathbf{v}$.

\section{RESULTS AND DISCUSSION}

\section{A. Vortex pinch-off}

In Fig. 1 we present contour plots of the vorticity field at three separate instants. The growing vortex ring is shown in its early stages of development in Fig. 1(a) (formation time $=1.5$ ). At a formation time of 4.1, the ring's growth has ceased, and a trailing shear layer carrying significant vorticity has developed [Fig. 1(b)]. Figure 1(c) shows that at a formation time of 8.5 , the vortex ring has separated from the trailing shear layer and advected away from it by selfinduction. These results are in agreement with those reported by Gharib et al. ${ }^{1}$ for large $L / D$ ratios (see their Fig. 5).

To further study the pinch-off process, we computed the forward-time FTLE fields from the particle image velocimetry data with an integration time of $T=3.3 \mathrm{~s}$ (or $\bar{U}_{p} T / D$ $=7.3$ ), where time $t_{0}=0$ corresponds to the initiation of fluid ejection from the cylinder. Figure 2(a) shows a contour plot of the forward-time FTLE field at the arbitrary time $t_{0}$ $=1.3 \mathrm{~s}$ (formation time $=2.8$ ) before the vortex ring pinches off. In Fig. 2(b), the locus of points on the LCS has been superimposed on the FTLE field, and points where the rate of strain is negative have been colored black while regions of

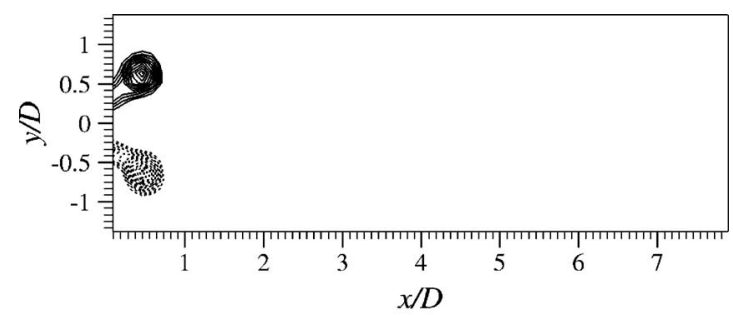

(a)

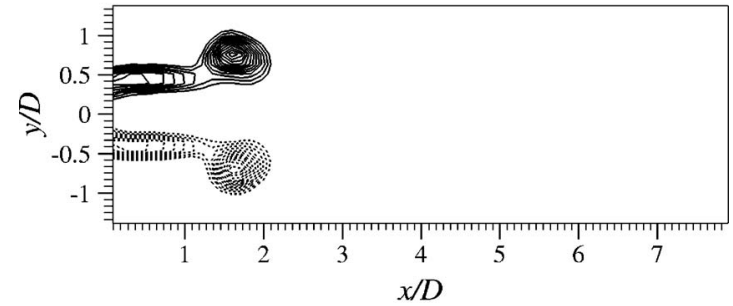

(b)

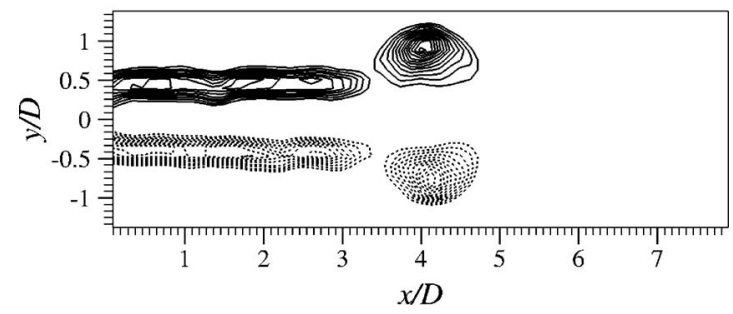

(c)

FIG. 1. Contours of vorticity at formation times: (a) 1.5, (b) 4.1, and (c) 8.5 . Contour levels: $\min =2 \%$ of $\omega_{\max }, \max =95 \%$ of $\omega_{\max }$, increment $=0.1 \%$. Dashed lines indicate negative vorticity. The cylinder exit plane is located in the $x / D=0$ plane, and the $x$-axis coincides with the vortex generator centerline. Flow is from left to right.

positive strain rate have been colored white. The spiral regions that correspond to the vortex ring core show alternating patterns of positive and negative strain rates, consistent with the shearing rotational flow within them. ${ }^{25}$ Upstream of the spiral regions, the strain rates normal to the LCS are positive, indicating that the ridge does indeed represent a hyperbolic repelling line.

As fluid continues to be ejected out of the cylinder, the vortex ring convects downstream by self-induction and the LCS lengthens until, at a formation time of $4.1\left(t_{0}=1.9 \mathrm{~s}\right)$, there is an abrupt change in the structure of the LCS. A new ridge, discontinuous from the original one, appears and the first LCS ends on the upstream end a few instants later. Figure 3 shows a contour plot of the FTLE field at a formation time of $4.5\left(t_{0}=2.1 \mathrm{~s}\right)$. The presence of a new ridge just downstream of the cylinder exit plane is evident in this figure, which corresponds to an instant of $0.2 \mathrm{~s}$ ( 0.43 formation time units) after the first appearance of the second ridge. The termination of the first LCS is visible a few instants later, and is evident in Fig. 4, taken at a formation time of 5.9.

At a formation time of $4.7\left(t_{0}=2.1 \mathrm{~s}\right)$, a third disconnected ridge appears just downstream of the cylinder exit, and the second LCS ends several time steps later. Figure 4 shows a contour plot of the FTLE field at a formation time of $5.9\left(t_{0}=2.7 \mathrm{~s}\right)$. The presence of a new ridge just downstream of the cylinder exit plane is evident in this figure, which 


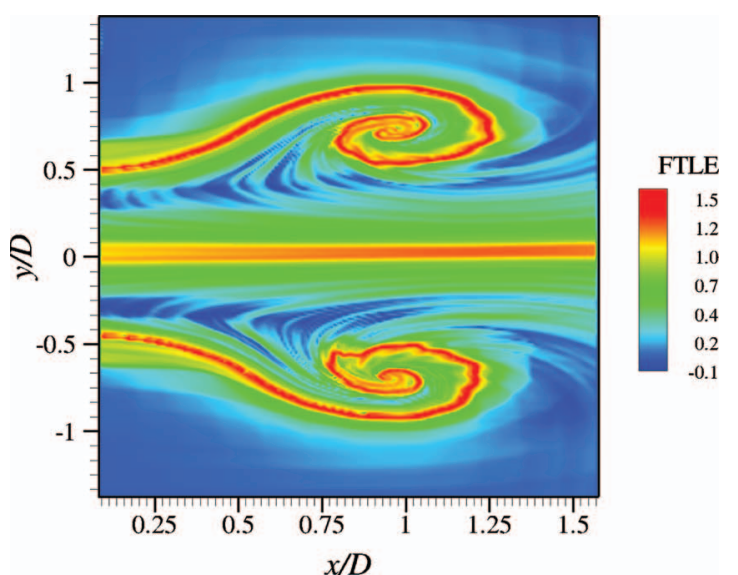

(a)

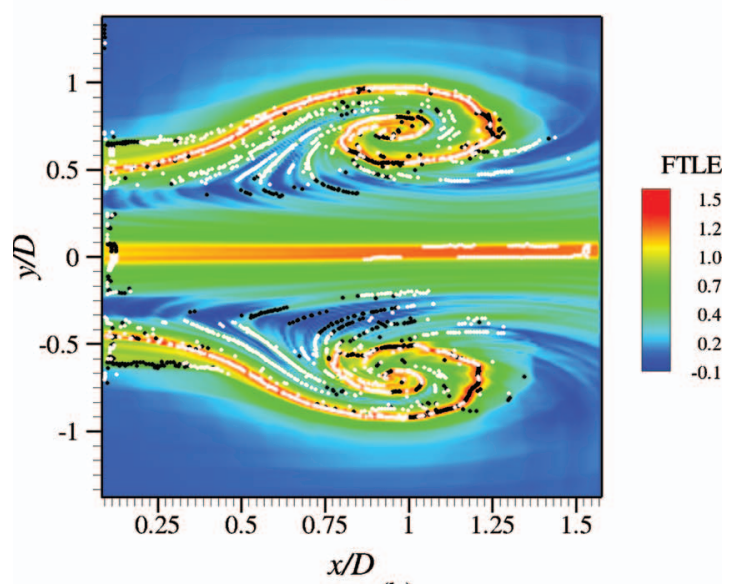

(b)

FIG. 2. (Color) Contours of the FTLE field at formation time of 2.8. In (b) the strain rate normal to the LCS has been superimposed. Positive strain rate is shown in white, negative in black.

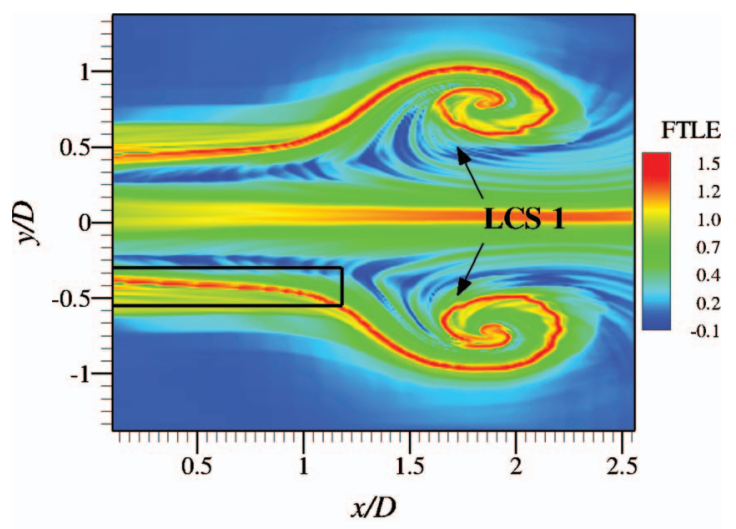

(a)

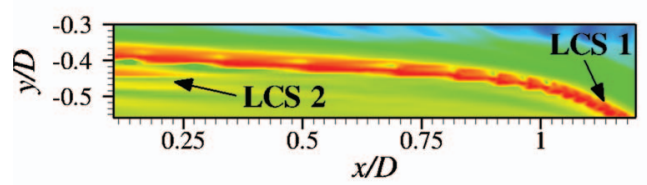

(b)

FIG. 3. (Color) Contours of the FTLE field at formation time of 4.5. A detail of the boxed region in (a) is shown in (b). A new LCS has formed and is indicated in (b) by an arrow.

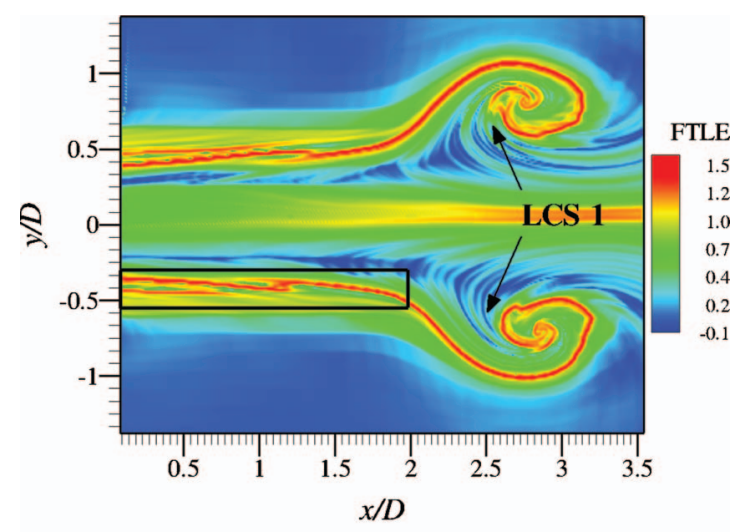

(a)

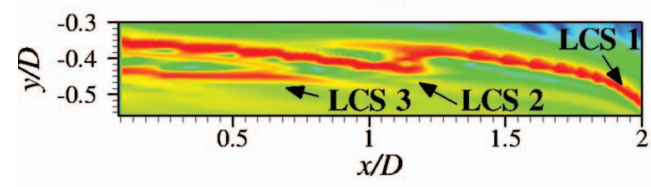

(b)

FIG. 4. (Color) Contours of the FTLE field at formation time of 5.9. A detail of the boxed region in (a) is shown in (b). LCSs 1-3 are indicated by arrows in (b). A new LCS (LCS 3) has just formed, and the termination of LCS 1 after the appearance of LCS 2 is also evident at this time.

corresponds to an instant of $0.5 \mathrm{~s} \mathrm{(1.1} \mathrm{formation} \mathrm{time} \mathrm{units)}$ after the first appearance of the third ridge. The termination of the second LCS is not evident until several time steps after the appearance of the third ridge, but it is clearly visible in Fig. 7.

Following Gharib et al., ${ }^{1}$ we computed the total circulation emanating from the piston-cylinder apparatus by integrating the vorticity contained within the lowest detectable contours. The circulation in the leading vortex ring was measured for large formation times, where the separation between the vorticity contours of the ring and those of the wake was clear. Figure 5 shows the nondimensional total circulation $\left(\Gamma / \bar{U}_{p} D\right)$ and vortex ring circulation as a function of formation time. Gharib et al. ${ }^{1}$ found that for large $L / D$ ratios the vortex ring circulation increased in a steplike fashion

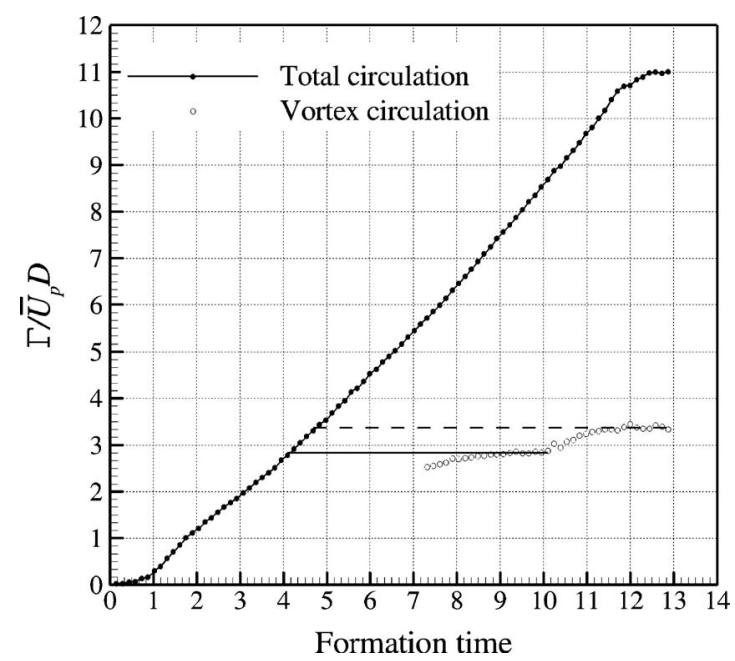

FIG. 5. Nondimensional total circulation and vortex ring circulation as a function of formation time. 
after long formation times (see their Fig. 10). This steplike increase was caused by the leading vortex in the trailing wake (hereafter referred to as vortex 2) catching up to and coalescing with the original vortex ring (hereafter referred to as vortex 1). ${ }^{1,26-28}$

In Fig. 5 we observe a similar steplike behavior after formation times of $\approx 10$. For formation times between $\approx 8$ and $\approx 10$, the vortex ring circulation is constant at $\Gamma / \bar{U}_{p} D$ $=2.8 \pm 0.1$. A straight line in Fig. 5 shows that at a formation time of approximately 4.2 the total circulation level equals that of vortex 1 for formation times between $\approx 8$ and $\approx 10$. The formation number is hence $4.2 \pm 0.2$, which agrees well with the appearance of the second LCS. A second straight line in Fig. 5 shows that at a formation time of $4.6 \pm 0.2$ the total circulation equals the final vortex ring circulation (i.e., the circulation of the combined vortices 1 and $2, \Gamma / \bar{U}_{p} D$ $=3.4 \pm 0.1$ ), meaning that vortex 2 must have itself pinched off from the trailing jet at this formation time. The initiation of pinch-off of vortex 2 at a formation time of $4.6 \pm 0.2$ corresponds well with the appearance of a third LCS at a formation time of 4.7 .

\section{B. Vortex pairing}

Observing the continued evolution of the LCS reveals the appearance of additional, disconnected LCS at later times. The location of these LCSs corresponds to the location of additional vortex rings developing in the trailing shear layer, so that the development of each new LCS provides insight into the evolution of vortices in the trailing wake. As new LCSs develop, many of them begin to roll up into spirals, and their structure begins to resemble that of the vortex ring core in the first LCS.

However, some of the new LCSs do not develop fully into tight spirals consistent with the formation of a new vortex ring, but rather merge with the preceding LCS. Figure 6(a) shows the first and second LCSs intersecting and beginning to merge. Figure 6(b) shows that LCS 2 merges completely with the first LCS and eventually becomes indistinguishable from it. This process is indicative of the beginning of the merger of vortices 1 and 2 .

The third LCS (whose corresponding vortex we shall refer to as vortex 3) does, however, develop fully into a tight spiral. Figure 7(a) shows the third LCS rolling up into a spiral. Figure 7(b), on the other hand, shows the fourth LCS (corresponding to vortex 4) intersecting with the third LCS. This intersection persists in time and is indicative of the early stages of the merger of the third and fourth LCSs, a process akin to the merging of the first and second LCSs in Fig. 6(b). The merging of the third and fourth LCSs is indicative of the beginning of the merger of vortices 3 and 4 .

Continued observation of the evolution of the emerging LCSs reveals a consistent "pairing" of adjacent vortices in the trailing jet. This phenomenon of vortex ring pairing in circular jets is well documented in the literature of turbulent mixing layers ${ }^{8,29,30}$ and is believed to be caused by wave instabilities in the vortex rings. For a description of the vortex pairing process and its causes, see Ref. 29.

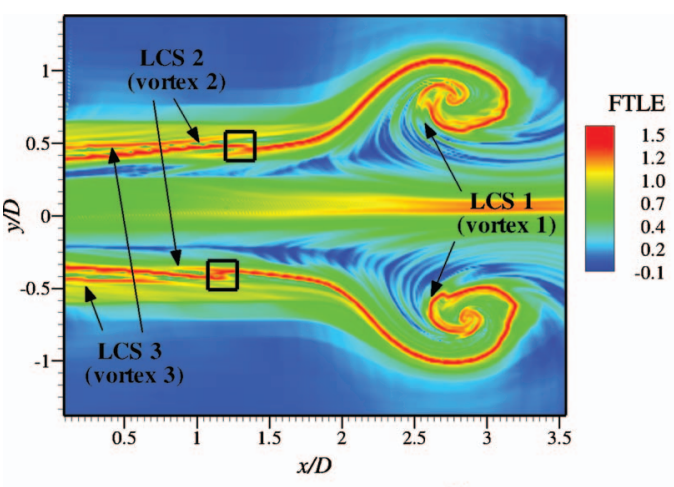

(a)

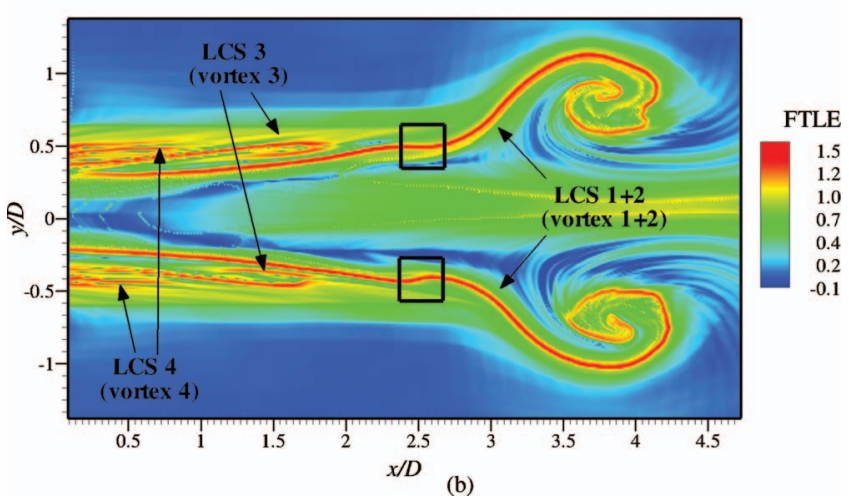

FIG. 6. (Color) Contours of the FTLE field. (a) Formation time=6.7. The boxed regions show the initial stages of the merger of LCSs 1 and 2. (b) Formation time $=8.0$. The first and second LCSs have merged at the regions indicated by the boxes.

Although the appearance of new disconnected LCS is a subtle feature, the new LCSs are persistent in time. The second LCS remains apparent until the merger of vortices 1 and 2 , at a formation time of 6.7, when it disappears as the LCSs corresponding to these vortices merge into a single coherent structure. In contrast, the third LCS remains separate from LCS 2 until the jet has shut off completely, since vortex 3 does not merge with the vortex ahead of it. Subsequent LCSs remain disconnected from their neighbors either until they disappear due to the merger of two vortices and their corresponding LCS, or until fluid has ceased to exit the nozzle.

The FTLE field is not an instantaneous measure of fluid particle separation, but rather an indication of the divergence of nearby trajectories over the integration time $T$. As a result, Haller ${ }^{23}$ showed that LCSs are robust to local anomalies in the velocity field, or short-lived features which have little or no effect on the overall flow. The observed appearances of new LCSs are therefore robust indicators of a physical change in the underlying vortex structures, as also found by Green et al. ${ }^{17}$ The appearance of a new LCS and termination of an existing one is the result of the separation of two distinct regions of vorticity-carrying fluid, which results in the formation of regions with separate dynamics. The merger of two LCSs into a single coherent structure, on the other hand, is indicative of the merger of two regions of vorticity into a single vortex.

\section{Comparison to other criteria}

Figure 1(b) shows a contour plot of the vorticity field at the initiation of pinch-off (formation time=4.1). Although 


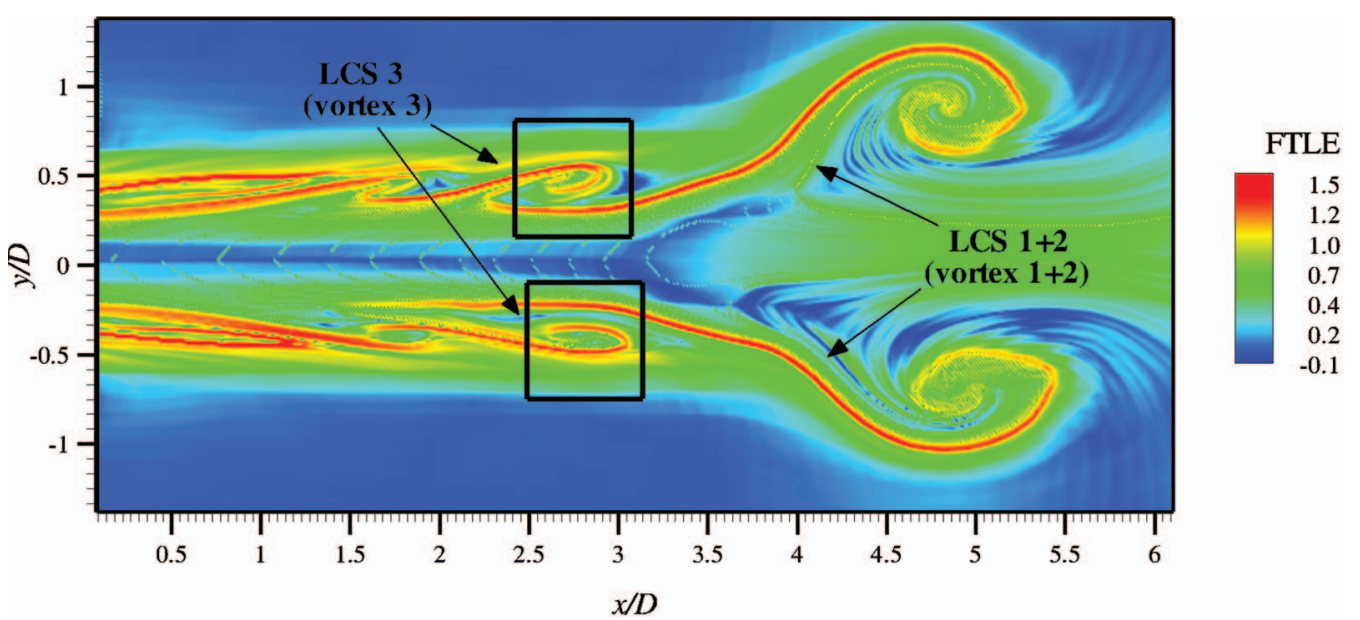

(a)

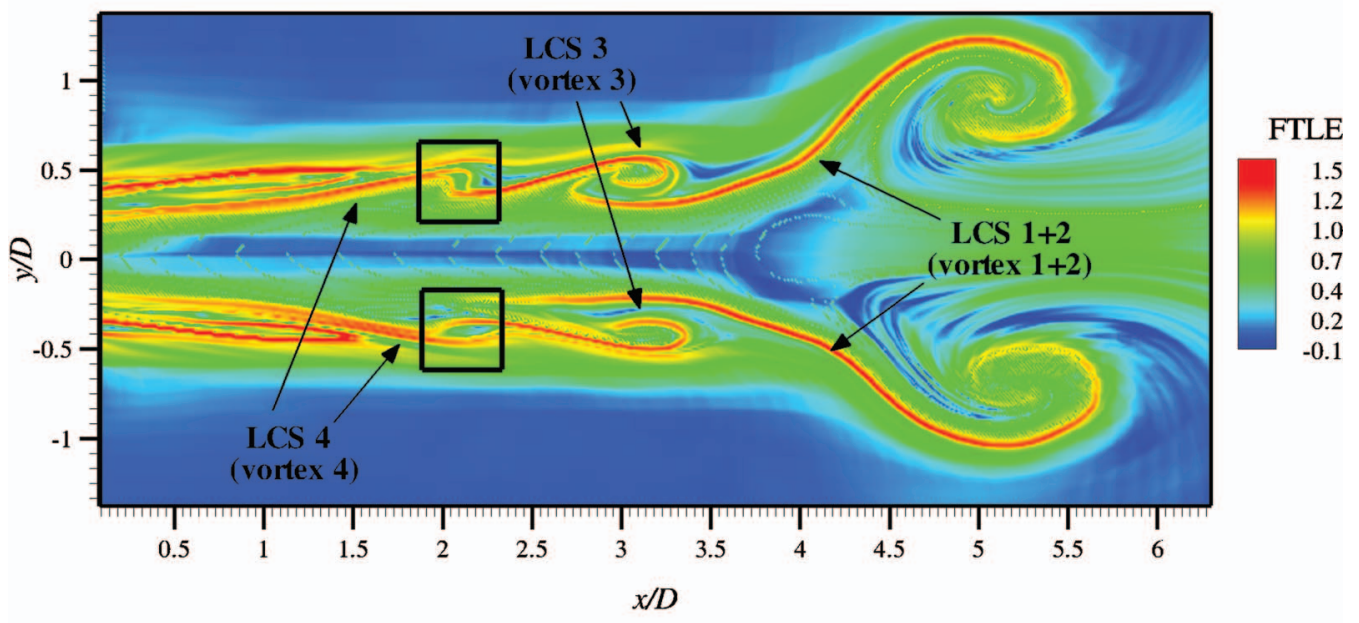

(b)

FIG. 7. (Color) Contours of the FTLE field. (a) Formation time=9.8. The boxed regions show the third LCS beginning to curl into a spiral. (b) Formation time $=10.2$. The third and fourth LCSs are merging at the regions indicated by the boxes. In both (a) and (b), the termination of LCS 2 (now merged with LCS 1) several time steps after the appearance of LCS 3 is evident.

the vortex ring ceases to accept vorticity at this time, it has not yet advected away from its trailing shear layer. A clear separation between vorticity contours of ring and wake is not evident until more than four formation time units later [Fig. $1(\mathrm{c})]$, and hence vorticity contours alone are insufficient to identity the initiation of pinch-off.

The vorticity field, however, can be integrated to obtain the circulation and apply the criterion of Gharib et al. ${ }^{1}$ Using this criterion, Krueger et al., ${ }^{12}$ Dabiri and Gharib, ${ }^{13}$ and others ${ }^{14-16}$ were able to determine the formation number for a variety of flow configurations. As discussed previously in Sec. III A, our results are in excellent agreement with the circulation method proposed by Gharib et al. ${ }^{1}$ Moreover, unlike the circulation criterion of Gharib et al., ${ }^{1}$ the current Lagrangian method is "instantaneous:" the initiation of pinch-off is evident from the forward FTLE field corresponding to the instant when it occurs. This does not imply, however, that the need for recording data past pinch-off is eliminated, but knowledge of only velocity field is required. This is of particular relevance in many real flows of biological interest, where the vorticity field breaks down and diffuses due to viscous effects, ${ }^{4}$ and criteria based on circulation prove difficult to use.

Like LCSs, instantaneous streamlines can be derived from the velocity fields alone and have been previously used to approximately describe vortex ring flows. ${ }^{18}$ Figure 8 shows the instantaneous streamlines of the flow computed in the laboratory reference frame. We do not present the streamlines in the reference frame of the leading vortex ring, unlike Dabiri and Gharib, ${ }^{18}$ since the leading ring and trailing jet are known to translate with different velocities. ${ }^{31}$ Since all of the flow structures do not translate at the same speed, a Galilean transformation to the frame of reference of the vortex ring cannot be applied.

In Fig. 8, the streamline patterns before the initiation of pinch-off [Fig. 8(a)], as pinch-off is initiated [Fig. 8(b)], and after the completion of the pinch-off process [Fig. 8(a)] are at formation times of 2.2, 4.1, and 8.7, respectively. There is no salient feature in Fig. 8(b) to indicate the initiation of 


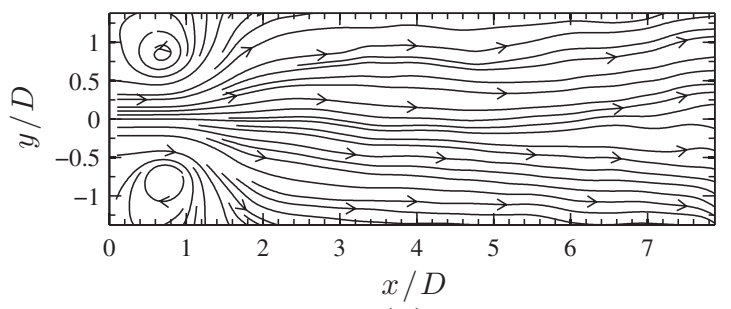

(a)

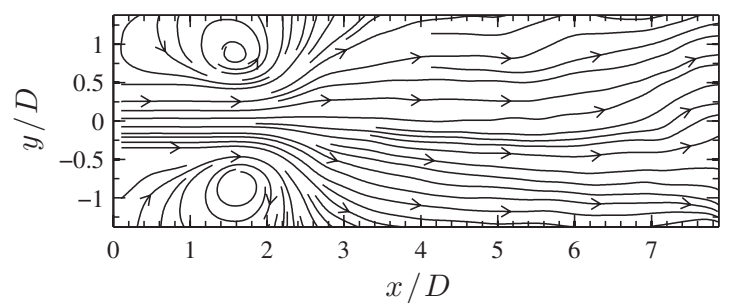

(b)

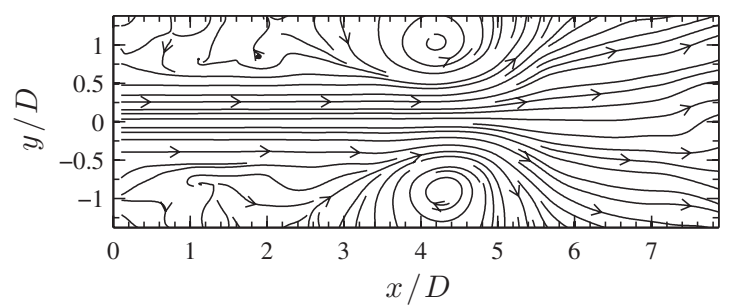

(c)

FIG. 8. Instantaneous streamlines at formation times: (a) 2.2, (b) 4.1, and (c) 8.7 .

pinch-off, and moreover there are no significant qualitative differences between the streamline patterns in Figs. 8(a)-8(c).

A variety of Lagrangian metrics also yields relatively little insight into the dynamics of pinch-off. Figure 9 shows the paths traced by 20 particles released at the nozzle exit plane as the jet was initiated. Figures 9(a)-9(c) show the pathlines before pinch-off, at the onset of pinch-off, and after pinch-off. Figure 10 shows streaklines starting at ten different locations on the nozzle exit plane before [Fig. 10(a)], at the onset of [Fig. 10(b)], and after pinch-off [Fig. 10(c)]. Finally, Fig. 11 shows the timelines of the flow at points before [Fig. 11(a)], at the initiation of [Fig. 11(b)], and after pinch-off [Fig. 11(c)]. There are no salient features in Figs. 9(b), 10(b), and 11(b) to reveal that the pinch-off process has been initiated. Moreover, careful comparison of the three parts in each figure does not suggest that either pathlines, streaklines, or timelines give insight into the pinch-off process.

While none of these Lagrangian metrics are particularly effective at identifying pinch-off, LCSs are an effective metric because they are a quantitative indicator of relative Lagrangian trajectories over time. LCSs identify regions of high particle separation and act as separatrices between regions of qualitatively different flows, ${ }^{11,23}$ and are thus suited for identifying the separation of different regions of vorticity-carrying flow that occurs during pinch-off. Furthermore, instantaneous streamlines, like streaklines and other Lagrangian metrics, are frame dependent and thus not suited

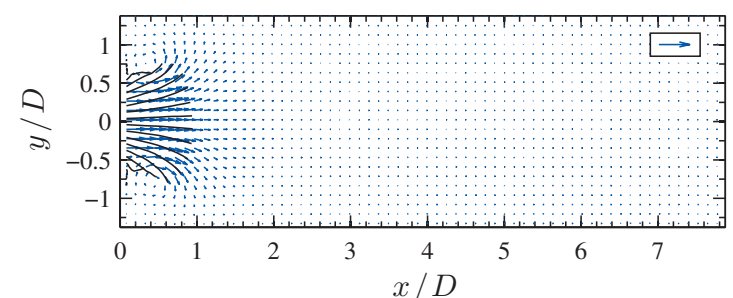

(a)

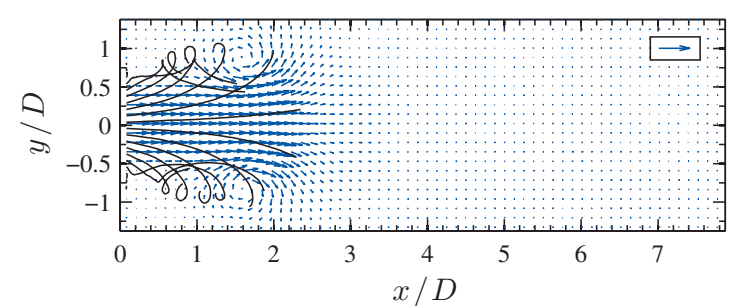

(b)

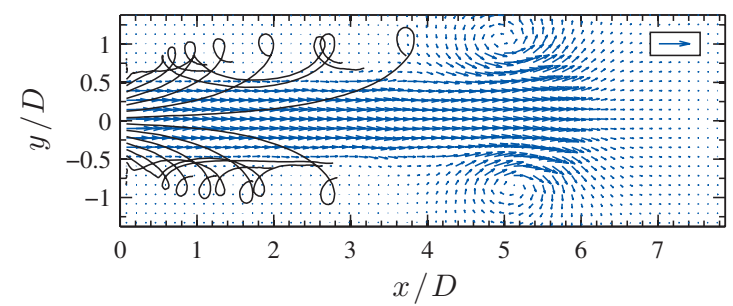

(c)

FIG. 9. (Color) Pathlines for 20 particles released at formation time $=0$ at formation times of (a) 1.5, (b) 4.1, and (c) 10.0. The velocity vector field is shown in the background for reference. The boxed arrows represent a speed of 4 nozzle diameters per second $(D / s)$.

for robust identification of the vortex structure in unsteady flows. ${ }^{11,24}$ In these cases, robust vortex identification requires knowledge of the time history of the motion, ${ }^{32}$ information that is built into the definition of the frame-independent FTLE.

\section{CONCLUSIONS}

A Lagrangian criterion for identifying vortex ring pinchoff in axisymmetric jet flow was proposed and demonstrated on a starting jet with $L / D \approx 12$. The appearance of a new LCS, disconnected from the original one, and the subsequent termination of the original LCS were found to coincide with the initiation of pinch-off. The new LCSs were found to either roll up into spirals, indicating the appearance of persistent vortices, or merge with the preceding LCS, indicating the merger of vortices in the shear layer. Hence, the appearance of a new LCS was found to be indicative of the initiation of vortex pinch-off and used to determine the formation number. Results obtained using the LCS criterion were compared with those obtained using the circulation criterion of Gharib et $a .^{1}{ }^{1}$ and were found to be in good agreement.

Furthermore, whereas Gharib et al. ${ }^{1}$ and others using similar methods recorded and identified the merging of the detached vortex ring with the leading vortex in its trailing wake, the LCSs revealed a consistent pattern of vortex pairing that extends beyond these first and second vortices. This continuous vortex pairing pattern has been identified as a 


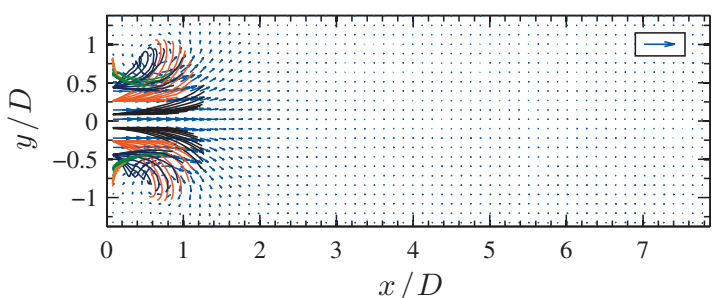

(a)

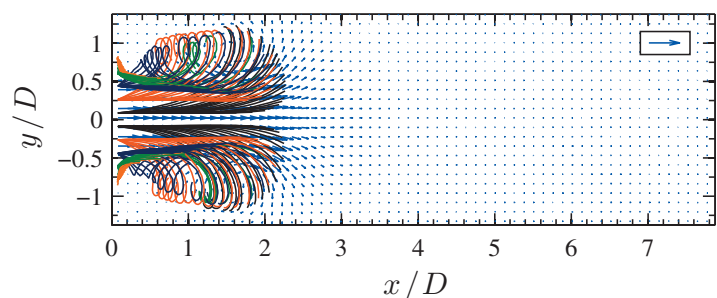

(b)

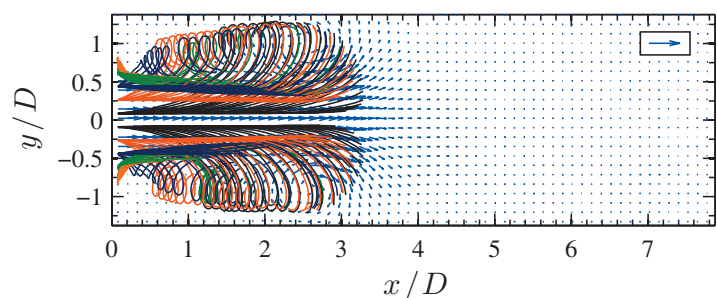

(c)

FIG. 10. (Color) Streaklines originating ten different points on the nozzle exit plane at formation times of (a) 2.2, (b) 4.1, and (c) 5.9. The velocity vector field is shown in the background for reference. The boxed arrows represent a speed of 4 nozzle diameters per second $(D / s)$.

recurrent feature in turbulent circular jets by various hot wire studies, ${ }^{8,29,30}$ yet it is difficult to observe from the velocity and vorticity fields alone. The LCSs, on the other hand, allow for clear identification of the pairing process from particle image velocimetry data.

Other pinch-off identification criteria, both Eulerian (vorticity contours, instantaneous streamline patterns) and Lagrangian (pathlines, streaklines, and timelines), were considered but found to yield little or no insight into the dynamics of pinch-off. The proposed LCS criterion therefore proved a superior identifier of pinch-off for the flow considered. Further, because the proposed LCS criterion is frame independent, independent of velocity derivatives, and robust to anomalies in the velocity field, it has the potential to prove a robust identifier of pinch-off in a wide variety of flows.

In particular, the proposed LCS criterion may prove to be a more suitable tool for characterizing unsteady and low Reynolds number biological flows, such as those generated by jetting swimmers ${ }^{3,4}$ or in the human heart. ${ }^{5}$ Future work will focus on applying the proposed criterion to such flows to determine the suitability of the criterion and its robustness.

The applicability of the proposed LCS criterion for identifying the onset of vortex pinch-off may also extend beyond the circular jets under consideration to nonaxisymmetric vortex-shedding flows. The application of this Lagrangian approach to characterizing the $2 \mathrm{~s}-2 \mathrm{p}$ transition in cylinder cross flow, ${ }^{33}$ for example, would be of interest.

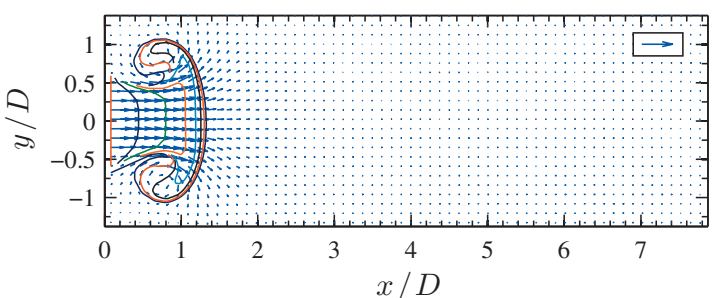

(a)

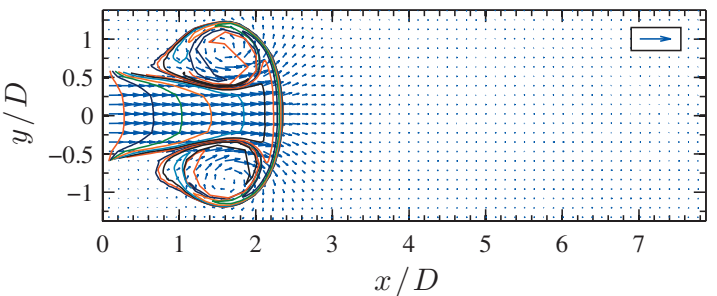

(b)

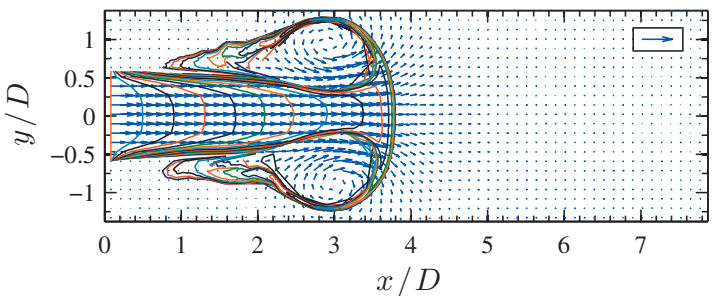

(c)

FIG. 11. (Color) Timelines at formation times: (a) 2.2, (b) 4.1, and (c) 6.6 The velocity vector field is shown in the background for reference. The boxed arrows represent a speed of 4 nozzle diameters per second $(D / s)$.

The proposed Lagrangian approach to vortex pinch-off is of particular interest in conjunction with the concept of "optimal" vortex formation. Krueger and Gharib ${ }^{2}$ found that the normalized average thrust produced during a jet pulse is maximized by jets with $L / D$ ratios just slightly greater than the observed formation number. This suggests the existence of an "optimum" $L / D$ ratio for vortex ring formation which occurs at the pinch-off point. The existence of this optimum bears intriguing implications for naturally occurring flows, since it poses the questions of whether the ejection of blood through the mitral valve in a healthy human heart is indeed optimal or whether the swimming and flying motions of organisms are tuned for optimal vortex formation. This question has been previously addressed, ${ }^{4,5,34-36}$ but the proposed LCS analysis has the potential to provide a more robust pinch-off identification criterion to aid in future studies of optimal vortex formation in complex biological flows.

Finally, it should be reiterated that although the LCS criterion is instantaneous in the sense that the LCS signature of the pinch-off process is evident at the initiation of pinchoff, it does not eliminate the need for recording forward-time data past pinch-off. Computing the forward FTLE field requires integrating particle trajectories in forward time, and thus requires recording the velocity field after pinch-off. However, the LCS criterion is dependent only on the forward-time velocity field, whereas criteria based on vortex circulation rely on the vorticity field. So although the need for forward-time data persists, the LCS metric eliminates the 
dependence on velocity derivatives, which become increasingly noisy during vortex breakdown.

\section{ACKNOWLEDGMENTS}

This work was conducted with support from the NSF programs in Fluid Dynamics and Biological Oceanography.

${ }^{1}$ M. Gharib, E. Rambod, and K. Shariff, J. Fluid Mech. 360, 121 (1998).

${ }^{2}$ P. S. Krueger and M. Gharib, Phys. Fluids 15, 1271 (2003).

${ }^{3}$ J. O. Dabiri, S. P. Colin, J. H. Costello, and M. Gharib, J. Exp. Biol. 208, 1257 (2005).

${ }^{4}$ I. K. Bartol, P. S. Krueger, W. J. Stewart, and J. T. Thompson, J. Exp. Biol. 212, 1506 (2009).

${ }^{5}$ M. Gharib, E. Rambod, A. Kheradvar, D. J. Sahn, and J. O. Dabiri, Proc. Natl. Acad. Sci. U.S.A. 103, 6305 (2006).

${ }^{6}$ J. S. Turner, J. Mech. Eng. Sci. 2, 96 (1960).

${ }^{7}$ K. Shariff and A. Leonard, Annu. Rev. Fluid Mech. 24, 235 (1992).

${ }^{8}$ A. J. Yule, J. Fluid Mech. 89, 413 (1978).

${ }^{9}$ T. Maxworthy, J. Fluid Mech. 64, 227 (1974).

${ }^{10}$ K. Shariff, A. Leonard, and J. H. Ferziger, Phys. Fluids 18, 047104 (2006).

${ }^{11}$ S. C. Shadden, J. O. Dabiri, and J. E. Marsden, Phys. Fluids 18, 047105 (2006).

${ }^{12}$ P. S. Krueger, J. O. Dabiri, and M. Gharib, J. Fluid Mech. 556, 147 (2006).

${ }^{13}$ J. O. Dabiri and M. Gharib, Phys. Fluids 16, L28 (2004).

${ }^{14}$ D. Jeon and M. Gharib, J. Fluid Mech. 519, 161 (2004).

${ }^{15}$ M. Milano and M. Gharib, J. Fluid Mech. 534, 403 (2005).
${ }^{16}$ M. J. Ringuette, M. Milano, and M. Gharib, J. Fluid Mech. 581, 453 (2007)

${ }^{17}$ M. A. Green, C. W. Rowley, and A. J. Smits, Chaos 20, 017510 (2010).

${ }^{18}$ J. O. Dabiri and M. Gharib, J. Fluid Mech. 511, 311 (2004).

${ }^{19}$ C. E. Willert and M. Gharib, Exp. Fluids 10, 181 (1991).

${ }^{20}$ G. Haller, Chaos 10, 99 (2000).

${ }^{21}$ G. Haller and G. Yuan, Physica D 147, 352 (2000).

${ }^{22}$ G. Haller, Physica D 149, 248 (2001).

${ }^{23}$ G. Haller, Phys. Fluids 14, 1851 (2002).

${ }^{24}$ M. Mathur, G. Haller, T. Peacock, J. E. Rupert-Felsot, and H. L. Swinney, Phys. Rev. Lett. 98, 144502 (2007).

${ }^{25}$ M. A. Green, C. W. Rowley, and G. Haller, J. Fluid Mech. 572, 111 (2007).

${ }^{26}$ W. Zhao, S. H. Frankel, and L. G. Mongeau, Phys. Fluids 12, 589 (2000)

${ }^{27}$ T. Maxworthy, J. Fluid Mech. 51, 15 (1972).

${ }^{28}$ Y. Oshima, T. Kambe, and S. Asaka, J. Phys. Soc. Jpn. 38, 1159 (1975).

${ }^{29}$ R. A. Petersen, J. Fluid Mech. 89, 469 (1978).

${ }^{30}$ A. K. M. F. Hussain and K. B. M. Q. Zaman, J. Fluid Mech. 101, 493 (1980).

${ }^{31}$ M. Shusser and M. Gharib, Phys. Fluids 12, 618 (2000).

${ }^{32}$ H. J. Lugt, Vortex Flow in Nature and Technology (Krieger, Malabar, Florida, 1995).

${ }^{33}$ C. H. K. Williamson and A. Roshko, J. Fluids Struct. 2, 355 (1988).

${ }^{34}$ P. F. Linden and J. S. Turner, Proc. R. Soc. London, Ser. B 271, 647 (2004)

${ }^{35}$ J. O. Dabiri, S. P. Colin, and J. H. Costello, J. Exp. Biol. 209, 2025 (2006).

${ }^{36}$ J. O. Dabiri and M. Gharib, Proc. R. Soc. London, Ser. B 272, 1557 (2005) 